\title{
PREFACE
}

\section{Bio-based Technologies for Resource Recovery}

\author{
Selected papers from the $15^{\text {th }}$ IWA World Conference on Anaerobic Digestion in Beijing, China
}

\author{
Aijie Wang $(\bowtie)^{1,2}$, David Stuckey $(\bowtie)^{3}$ \\ 1 State Key Laboratory of Urban Water Resource and Environment, Harbin Institute of Technology (SKLUWRE, HIT), Harbin 150090, China \\ 2 Key Laboratory of Environmental Biotechnology, Research Center for Eco-Environmental Sciences, Chinese Academy of Sciences, \\ Beijing, 100085, China \\ 3 Department of Chemical Engineering, Imperial College London, SW7 2AZ London, UK*
}

(C) Higher Education Press and Springer-Verlag GmbH Germany, part of Springer Nature 2018

\begin{abstract}
Anaerobic digestion (AD) is an indispensable technology for mankind to live a sustainable life in this world. Currently, anaerobic technology is being increasingly adopted as a cost-effective way for waste(water) treatment, but has also evolved from an approach solely for waste(water) treatment to an integrated method for resource recovery, e.g. water, energy (biogas), nitrogen, phosphorous, etc. Implementation of anaerobic technology even carries associated benefits such as the mitigation of climate change. Hence, we are standing at a turning point in reframing the future of anaerobic technology, and move towards a more sustainable world. The demand for fossil energy substitution, nutrient recirculation and efficient waste management, is driving technical innovation strongly. Therefore, with the theme of "Towards a More Sustainable World", the AD-15 conference focused on the most promising anaerobic technologies, as well as emerging and cutting-edge techniques and ideas that can influence AD's future development. The conference was jointly organized by the International Water Association (IWA), Harbin Institute of Technology, Tsinghua University, and Research Center for Eco-Envrionmental Sciences, CAS. This conference was part of the IWA Anaerobic Digestion Specialist Group Conference series, which were conceived as an international forum targeting state-of-the-art anaerobic bioprocesses.

In this conference, more than 1,100 delegates from over 70 countries attended the meeting. Over 800 conference abstracts were submitted, focusing on 10 topics \& sessions, covering microbiome \& biotransformations, modeling, high solids $\mathrm{AD}$, innovative technology, agricultural waste, industrial \& municipal wastewater treatment, resource recovery, pre- \& post- treatment, and policy traction. Of which, 16 papers have been selected to publish in FESE as a special issue with the theme of Bio-based Technologies for Resource Recovery. The main topics include (i) innovative/emerging technologies for energy recovery, (ii) resource recovery from wastes or wastewater, (iii) case studies and practical use of waste-to-biofuels, and (iv) methodology on microbial culture and functional community characterization.

Thanks to the successful organization of the $15^{\text {th }}$ AD conference, we acknowledge all the sponsorship and financial support of the conference. We appreciate the efforts by all the organizers, and volunteers for the conference. For the special issue of FESE, we give our thanks to the authors, editors and the assistance from the Editorial Office of FESE. In particular, we appreciate the support of Professor Xia Huang, Executive Associate Editor-in-Chief of FESE, for agreeing to dedicate an issue to this conference. Also, we are grateful to Ms. Xiangyi Zhang, and Mr. Lizheng Wang in the Editoral Office for their kind help. We also wish to thank Dr Wenzong Liu and the reviewers for their precious time and efforts on the submitted paper to this special issue.
\end{abstract}

$\overline{\text { Received July 24, } 2018}$
$\overline{\text { E Corresponding authors }}$
E-mail: ajwang@rcees.ac.cn (A. Wang); d.stuckey@imperial.ac.uk (D. Stuckey)

${ }^{*}$ Special Issue - Bio-based Technologies for Resource Recovery (Responsible Editors: Aijie Wang \& David Stuckey) 